\title{
The 49th Symposium on Powder Technology
}

The 49th Symposium on Powder Technology was held at Senri Hankyu Hotel, Japan on Monday, September 7, 2015. It was organized by Hosokawa Powder Technology Foundation with the sponsorship of Hosokawa Micron Corporation. More than 160 people from the industries and universities attended this symposium. The theme of the symposium this year was "Powder Technology, advancing in response to the requirements of the era". There were six lectures with questions and answers followed by a get-acquainted party for further free discussions.

The first lecture was given by Prof.Otani, who was presented the KONA Award last year, on his lifework research in the field of air filters including the recent trends of new advanced filters. Four lectures were carried out by the professors with the diversity of the fields including the electrode/solid electrolyte composite of secondary batteries, the functional devices using 3D micro/nano stereolithograph and the tailored composite particles as well as its processing. The other two lectures were from the industrial fields introducing the casting technology, which has been developed since the ancient days supporting the manufacturing industry, and the new-type vacuum dryer with the agitating mechanism which is capable of handling heat-sensitive materials like pharmaceuticals and food staff.

The contents of the symposium are shown in the followings.

\section{The 49th Symposium on Powder Technology}

Theme: Powder Technology, advancing in response to the requirements of the era

Opening address Prof. emeritus Minoru Takahashi (Nagoya Institute of Technology)

Session 1 Chaired by Prof. emeritus Fumio Saito (Tohoku University)

- Lecture 1

"Present Status of Air Filters and Exploration of Their New Applications"

Prof. Yoshio Otani (Kanazawa University)

- Lecture 2

"Room Temperature Fabrication of Electrode-Solid Electrolyte Composite for All-Solid-State

Rechargeable Lithium Batteries"

Prof. Yasutoshi Iriyama (Nagoya University)

Session 2 Chaired by Prof. Hirofumi Takeuchi (Gifu Pharmaceutical University)

- Lecture 3

"Development of Functional Devices Using Three-Dimensional Micro/Nano Stereolithography" Prof. Shoji Maruo (Yokohama National University)

- Lecture 4

"Design of Tailored Composite Fine Particles and Its Processing"

Prof. Satoru Watano (Osaka Prefecture University) 
or

Session 3 Chaired by Prof. Makio Naito (Osaka University)

- Lecture 5

"Casting Technology, Supporting Manufacturing Industry over 5,000 Years"

Dr. Haruyoshi Hirano (Takeyama Foundry Co., Ltd.)

- Lecture 6

"New Dyer for the Efficient Drying of Heat-Sensitive Materials" Mr. Takashi Ochiai (Hosokawa Micron Corporation)

Closing address Mr. Yoshio Hosokawa (President of Hosokawa Powder Technology Foundation)

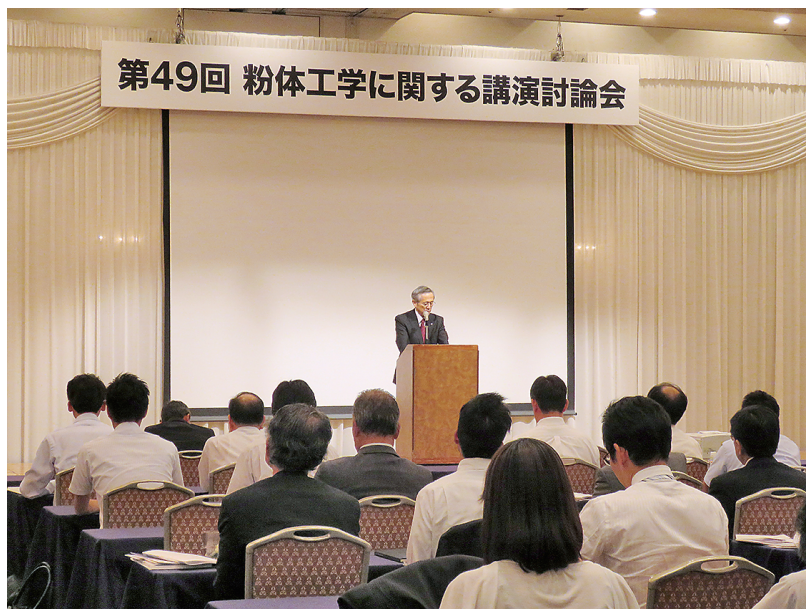

The 49th Symposium on Powder Technology

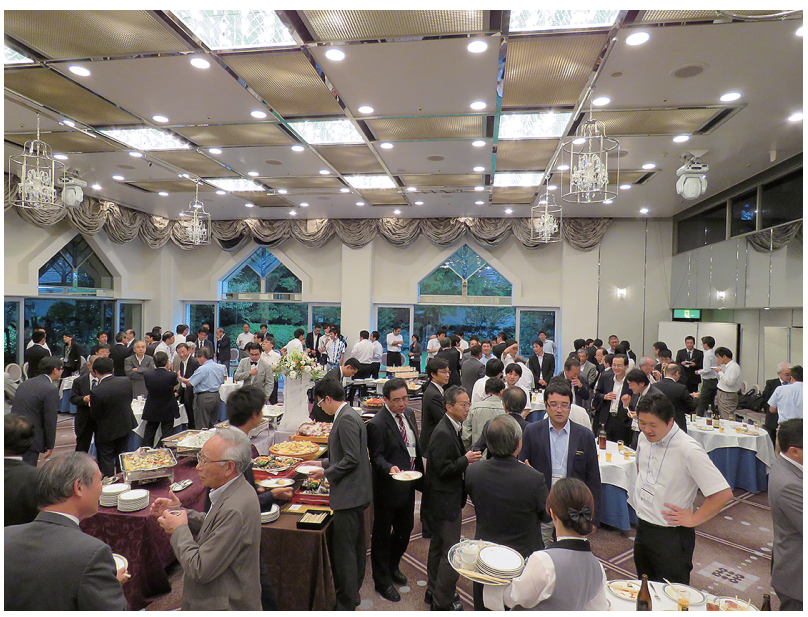

Get-acquainted Party 